\title{
MULTI-STAGE STOCHASTIC ELECTRICITY PORTFOLIO OPTIMIZATION IN LIBERALIZED ENERGY MARKETS
}

\author{
R. Hochreiter, ${ }^{1}$ G. Ch. Pflug, ${ }^{1}$ and D. Wozabal ${ }^{1}$ \\ ${ }^{1}$ Department of Statistics and Decision Support Systems, University of Vienna; Universitätsstraße \\ 519, 1010 Vienna, Austria, \{ronald.hochreiter,georg.pfug,david.wozabal\}@univie.ac.at
}

\begin{abstract}
In this paper we analyze the electricity portfolio problem of a big consumer in a multi-stage stochastic programming framework. Stochasticity enters the model via the uncertain spot price process and is represented by a scenario tree. The decision that has to be taken is how much energy should be bought in advance, and how large the exposition to the uncertain spot market, as well as the relatively expensive production with an own power plant should be. The risk is modeled using an Average Value-at-Risk (AVaR) term in the objective function. The results of the stochastic programming model are compared with classical fix mix strategies, which are outperformed. Furthermore, the influence of risk parameters is shown.
\end{abstract}

keywords: Stochastic Optimization, Scenario Generation, Energy Markets, Optimal Electricity Portfolios, Average Value-at-Risk

\section{Introduction}

In this paper, we present a multi-stage stochastic optimization model for calculating optimal electricity portfolios. We refer to [10] for an overview of stochastic programming and to [1] for applications to the energy market. The general formulation of a multistage stochastic optimization program is

$$
\begin{array}{ll}
\text { minimize } & \mathbf{E}(f(x(\xi), \xi)) \\
\text { subject to } & (x(\xi), \xi) \in \mathcal{X} \\
& x \in \mathcal{N}
\end{array}
$$

where $\xi$ denotes a multi-dimensional stochastic process describing the future uncertainty. The constraint-set $\mathcal{X}$ contains feasible solutions $(x, \xi)$ and the (non-anticipativity) set $\mathcal{N}$ of functions $\xi \mapsto x$ is necessary to ensure, that the decisions $x_{t}$ are only based on realizations up to stage $t\left(\xi_{0}, \ldots, \xi_{t}\right) . f(x(\xi), \xi)$ is some cost function.

Please use the following format when citing this chapter:

Author(s) [insert Last name, First-name initial(s)], 2006, in IFIP International Federation for Information Processing, Volume 199, System Modeling and Optimization, eds. Ceragioli F., Dontchev A., Furuta H., Marti K., Pandolfi L., (Boston: Springer), pp. [insert page numbers]. 
We apply this framework to the optimization of electricity portfolios. Additionally, an Average Value-at-Risk functional is included, enabling modern risk management, which is necessary to survive in liberalized energy markets economically.

This paper is organized as follows. Section 2 describes the estimation and simulation of the (uncertain) electricity spot market, which is the most import input for the stochastic program. Section 3 provides a detailed overview of the underlying model. Section 4 summarizes numerical results, while Section 5 concludes the paper.

\section{Scenario Generation}

\subsection{Estimation and simulation of the spot market}

The generation of scenarios for the possible development of spot prices is based on an econometric model which is designed to capture the past movements of the spot price as good as possible. This model will be capable of giving good estimates for the expected price at every hour of the period under consideration. To generate realistic scenarios we simulate the residuals of the model and thereby distort the prediction to get a possible trajectory for the spot price of energy. At the end, we compute the mean for 4 consecutive values and therefore reduce the price movement in one day to six data points, representing the average price from $0-4,4-8,8-12,12-16,16-20,20-24$ o'clock respectively.

The modeling of the spot prices is done using linear regression where the main explanatory variables are: the hour of the day, the day of the week, and the season. The regressors related to time are modeled in such a way that there are initially $24 \times 7 \times 3=504$ dummy variables indicating which hour of which day in what season a specific data point belongs to. Obviously, this yields to an unnecessary huge model, which can be reduced in a further step. The reduction is based on the observation that the coefficient of a dummy variable will be the mean of the data points that it points to. A feasible way to reduce the number of regressors would be to compare the means of the different hours on the different days in the different seasons and club two regressors if the means are only insignificantly different. To determine whether two means are different, we use the Kruskal-Wallis test (see for example [5]), since it is based on rank order and does not assume the data to be distributed according to any specific distribution. The necessity of such a non-parametric approach will become clear, when we discuss the residuals of the model. With this procedure, we are able to significantly reduce the number of regressors without sacrificing much of the accuracy in predicting the expected (mean) price in the respective hours.

As already mentioned, we also use temperatures as explanatory variables. This proved to be beneficial (in terms of explanatory power) and also supports the intuition of modeling temperatures not as a single variable, but to split it up 
into six variables measuring the effect of temperature on power prices - not for the whole day, but for the six 4-hour blocks described above.

This model of the stock prices focuses heavily on the demand side of the market, which is reasonable, since the average prices and the daily patterns of price movements can be explained with these factors pretty well (adjusted $\mathrm{R}^{2}=0.6$ ). To explain long term changes in the market and to understand the peaks in the hourly spot price, it would be a valuable idea to include the supply side too. This would allow for a better understanding of the energy market and probably boost the insample accuracy too. However, when it comes to simulation these gains would probably be lost, because the supply factors are hard to forecast.

To capture some of these effects we add an $\mathrm{AR}(1)$ and $\mathrm{AR}(25)$ term to our Regression model and obtain a $\mathrm{R}^{2}$ of 0.84 .

An inspection of the residuals shows, that those are clearly not normal. Since we need to simulate from the residual distribution, we have to fit some parametric models to the empirical residuals. It turns out that the distributions are extremely wide stretched (peaks in the prices) and are therefore heavy tailed. We chose to use a stable distribution to fit the data, since the family of stable distributions contains heavy tailed distributions too, and it is known that sums of independent identically distributed random variables follow some stable law. The family of stable distributions can be characterized through their characteristic function

$$
\mathbf{E}\left(e^{i u X}\right)=\left\{\begin{array}{cl}
e^{-\gamma^{\alpha} \mid u^{\alpha}\left(1+i \beta\left(\tan \frac{\pi \alpha}{2}\right) \operatorname{sign}(u)\left(|\gamma u|^{1-\alpha}-1\right)\right)+i \delta u} & , \alpha \neq 1 \\
e^{-\gamma|u|\left(1+i \beta \frac{2}{\pi} \operatorname{sign}(u) \log (\gamma|u|)\right)+i \delta u} & , \alpha=1
\end{array}\right.
$$

where $0<\alpha \leq 2$ can be interpreted as an index of stability (everything below 2 is heavy trailed), $\beta$ is a skewness parameter, $\gamma$ a scale parameter, and $\delta$ a location parameter. For a in depth description of heavy tailed distributions, their properties and how to fit them see [8]. To fit the stable distribution to our residuals, we split the residuals into 6 groups according to which time slot they belong to and separately estimate stable distributions for these time slots. In a next step we generate a sufficient number of random draws from these distributions and use those together with the predicted prices to obtain spot price trajectories. For fitting the stable distribution and the generation of the random variates we use the software stable.exe (see [6]).

For fitting the model we use one year of hourly data (01.06.2005-31.05.2006) from the European Energy Exchange (EEX). Using the fitted model and distribution of the residuals, we simulate price trajectories corresponding to a price development of half a year. Figure 1 dipicts the means of the simulated values in each of the 4 hour slots (left) and 20 days of a typical simulation trajectory (right). 

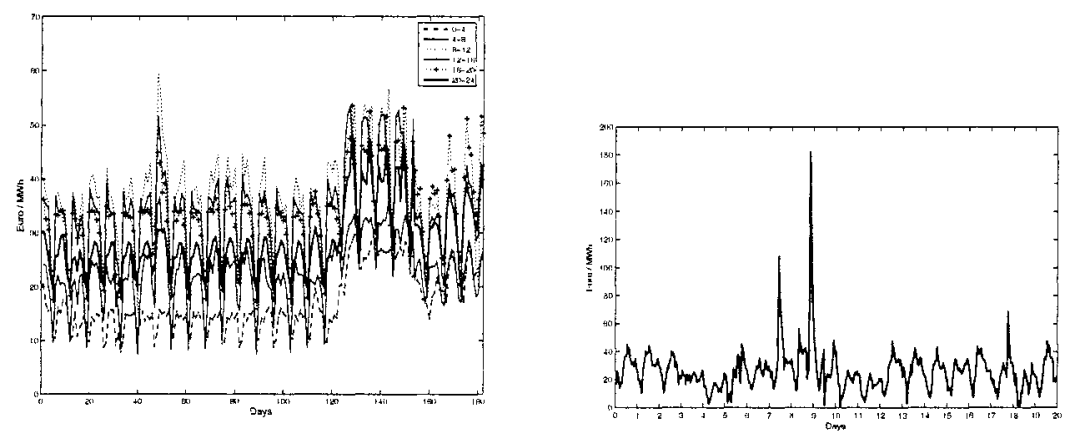

Figure 1. Averages of simulated price trajectories (left), Sample simulated spot price trajectory (right).

\subsection{Generation of scenario trees}

We apply a scenario generation method based on [7]. This method is optimization-problem related, and aims at minimizing a probability metric with a $\zeta$-structure, i.e. the uniform distances of expectations of functions taken from a class $\mathcal{H}$ of measurable functions. The Wasserstein distance (from the class $\mathcal{H}$ of Lipschitz continuous functions), which plays an important role for stability results and approximation of stochastic programming models, has been used.

The implemented method generates scenario trees with a stagewise-fixed structure, which differs from other methods, e.g. [2] or e.g. an explicit method for the energy market in [4].

The size of the generated underlying spot market scenario tree with the stagewise-fixed structure, which was used for numerical experiments, is

$$
n_{t}=[100,200,200+(t-2) \cdot 2],
$$

where $n_{t}$ denotes the number of nodes in stage $t$, resulting in 560 scenarios.

\section{Optimization Model}

The goal of the optimization is to determine the amount of energy that should be bought in advance for a time period of half a year. The driving factor of the optimization is the expected demand for energy at all points of the considered time period. This demand is assumed to be non-stochastic and can be met in three ways. One may

1 buy electricity on the spot market,

2 produce electricity, and/or 
3 buy supply contracts for future delivery of energy.

The supply contracts are designed as follows: every month the producer can consume a certain amount of energy $u_{i}$ in every of the six periods of the day. This amount is part of the contract and can be specified by the consumer. On every day the energy consumption is bounded by a fraction of the overall monthly consumption, of course with the additional restriction, that the monthly consumption adds up to the pre-specified amount.

On the tree described in section 2.2 the program is shown in equation (1), which minimizes the expected total cost and the (terminal) Average Valueat-Risk (AVaR). Let $A V @ R_{\alpha}$ be defined as the solution of the optimization problem

$$
\inf \left\{a+\frac{1}{1-\alpha} \mathbf{E}[Y-a]^{+}: a \in \mathbf{R}\right\},
$$

where $Y$ is a random cost variable. With a finite set of scenarios this optimization problem can be reformulated as a linear program (see [9]).

$$
\begin{array}{llll}
\operatorname{minimize} & \sum_{n \in \mathcal{N}^{(T)}} P_{s(n)} c_{n}^{(t)}+\kappa\left(\gamma+\sum_{n \in \mathcal{N}^{(T)}}\right. & \left.\frac{P_{s(n)} z_{n}}{1-\alpha}\right) \\
\text { subject to } & g_{n, h}+s_{n, h}+m_{n, h} & \geq D_{T(n), h} & (a) \\
& g_{n, h} & \leq G^{\max } & (a) \\
& m_{n, h} \xi_{n, h}+g_{n, h} C^{p r o d}+s_{n, h} \phi F_{T(n)}^{p} & =c_{n, h} & (b) \\
& m_{n, h} \xi_{n, h}+g_{n, h} C^{p r o d}+s_{n, h} \phi F_{T(n)}^{o} & =c_{n, h} & (c) \\
& s_{n, h} & \leq \beta u_{M(n), h}(a) \\
& \sum_{n \in \mathcal{N}_{i}} s_{n, h} & \leq u_{m, h} & (d) \\
& \sum_{t \in \mathcal{T}} c_{R(n, t), h} & =c_{n}^{(t)} & (e)
\end{array}
$$

$M(n)$ is the month of the node $\mathrm{n}, g_{n}, h, s_{n, h}, m_{n, h}$ electricity coming from own production (generation), the supply contract and the spot market in node $n$ and hour-block $h$ respectively, while $D_{n, h}$ denotes the (deterministic) demand. $\beta$ represent the daily upper constraint on consumption, defined as fraction of the overall monthly consumption.

$R(n, t)$ returns the scenario predecessor node of terminal node $n$ in stage $t$, $P_{s(n)}$ is the scenario probability of the scenario terminating in node $n$. $T(n)$ returns the stage of node $n, F_{t}^{p}, F_{t}^{o}$ are the costs of the peak and offpeak future at stage $t$, and $\phi$ is a factor by which the contract is cheaper than the future price. $u_{m, h}$ is the optimal contract volume for month $n$ and hour-block $h . \xi_{n, h}$ is the stochastic spot price.

The constraints the model (1) are defined for different parameter sets. The letter in parenthesis at the right indicates, which group of sets the constraint is defined for. Let $\mathcal{H}$ be the set of all hour-blocks, and $\mathcal{H}_{p}$ and $\mathcal{H}_{0}$ peak and off-peak hour-blocks. $\mathcal{N}$ the set of all nodes and $\mathcal{N}_{T}$ the set of all terminal 


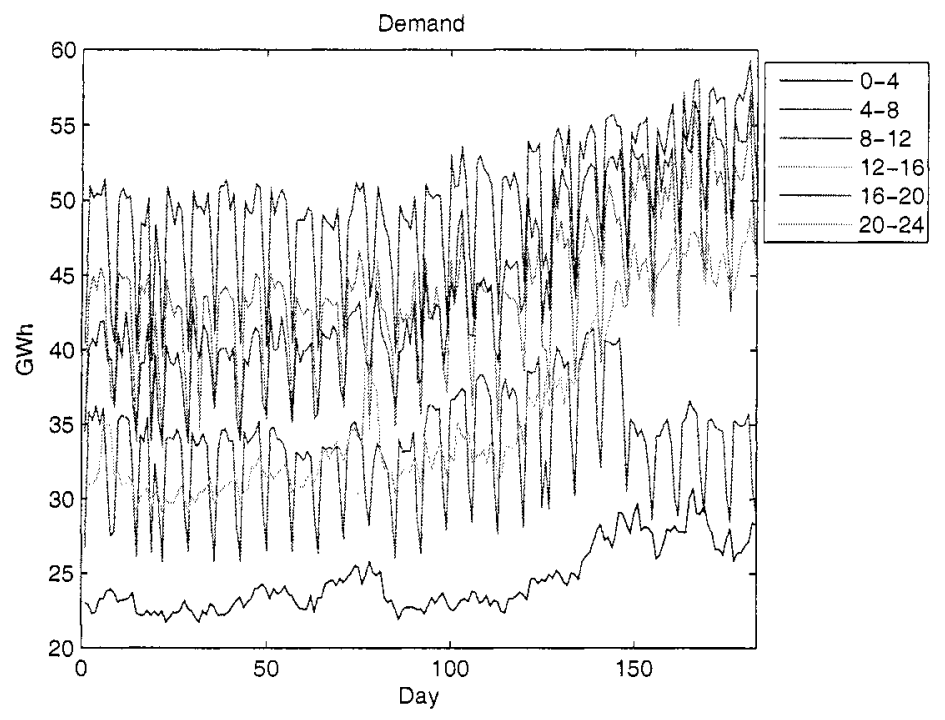

Figure 2. Demand of a large local energy distributor.

nodes. $\mathcal{M}_{m}$ is the set of all stages/nodes within month $m$. The following group of sets are applied:

(a) $\forall n \in \mathcal{N}, \forall h \in \mathcal{H}$,

(b) $\forall n \in \mathcal{N}, \forall h \in \mathcal{H}_{p}$,

(c) $\forall n \in \mathcal{N}, \forall h \in \mathcal{H}_{0}$,

(d) $\forall i \in \mathcal{M}_{m}, \forall m \in\{$ June, $\ldots$, November $\}, \forall h \in \mathcal{H}$.

(e) $\forall n \in \mathcal{N}_{T}, \forall h \in \mathcal{H}$.

\section{Numerical Results}

A (deterministic) demand forecast of a large local energy distributor has been used. This demand is shown in Figure 2 for each of the six 4-hour blocks described in Section 2.1.

To enable a numerical comparison, the following parameters have been fixed: The cost of producing energy $C^{\text {prod }}$ is Euro 70 and the maximum production $G^{\max }$ per 4-hour block is 5000MW. Factor $\beta$ has been set to 0.1 and $\phi$ to 0.9 . 


\begin{tabular}{cccc} 
Spot Market & Supply Contract & Production & Expected Total Cost (Euro) \\
\hline $20 \%$ & $60 \%$ & $20 \%$ & 1.838 .258 .100 \\
$20 \%$ & $70 \%$ & $10 \%$ & 1.706 .414 .546 \\
$20 \%$ & $80 \%$ & $0 \%$ & 1.574 .570 .991
\end{tabular}

Table 1. Expected Total Cost for Fix Mix Portfolios

\begin{tabular}{ccccccc} 
& June & July & August & September & October & November \\
\hline $0-4$ & 229153 & 546926 & 510129 & 449503 & 407692 & 802547 \\
$4-4$ & 208374 & 705748 & 423220 & 249749 & 197142 & 872110 \\
$8-12$ & 169746 & 624004 & 374384 & 133423 & 155421 & 869426 \\
$12-16$ & 253450 & 600427 & 287414 & 131299 & 187684 & 835807 \\
$16-20$ & 721441 & 1113900 & 923807 & 832003 & 595822 & 1262270 \\
$20-24$ & 582319 & 693024 & 216780 & 190338 & 386261 & 1376000
\end{tabular}

Table 2. Example contract for $\alpha=0.9$ (MWh)

\begin{tabular}{ccc}
$\alpha$ & Expected Total Cost (Euro) & $\sum$ Contracted Volume (GWh) \\
\hline 0.7 & 1.221 .079 .145 & 19990 \\
0.8 & 1.220 .568 .060 & 19637 \\
0.9 & 1.220 .026 .120 & 19118 \\
0.95 & 1.219 .763 .475 & 18747
\end{tabular}

Table 3. Aggregated results of the stochastic optimization for different risk parameters

\subsection{A Fix Mix Solution}

To compare the stochastic solution, we calculated some fix mix strategies. The results are shown in Table 1.

\subsection{The Stochastic Solution}

The stochastic optimization models have been implemented in AMPL (see [3]). The workflow has been developed in MatLab and some parsing scripts have been implemented in Python. The optimization problems have been solved with the MOSEK interior point solver.

The optimization problems were solved with a Pentium $4(2 \mathrm{GHz})$ with $1 \mathrm{~GB}$ RAM running Debian GNU/Linux. The average solution time of the underlying problems is half an hour.

A typical optimal contract volume sheet is shown in Table 2 for $\alpha=0.9$. To see the influence of the risk parameter $\alpha$, the expected total cost of the portfolio in Euro and the sum of contracted volume in GWh is shown in Table 3. Additionally, these results show that the stochastic solution clearly outperforms fix mix strategies. 


\section{Conclusion}

In this paper we proposed a model to solve the electricity portfolio problem of a big consumer in a multi-stage stochastic programming framework. The decision that has to be taken is how much energy should be bought in advance and how large the exposition to the uncertain spot market, and the relatively expensive production by an own power plant should be. It has been shown that the underlying spot price can be realistically estimated and simulated with a regression model. The underlying scenario trees representing the uncertain future spot prices are used to calculate optimal electricity portfolios. Different supply contract details have been included, such that the model is ready to be applied for practical usage.

The results show that the solution of the multi-stage stochastic program clearly outperforms classical fix mix strategies. Furthermore, by varying the risk parameter $\alpha$, the consumer can fine-tune his optimal decision.

\section{References}

[1] W. Römisch, A. Eichhorn and I. Wegner. Mean-risk optimization of electricity portfolios using multiperiod polyhedral risk measures. IEEE St. Petersburg Power Tech 2005 (to appear), 2005.

[2] J. Dupačová, N. Gröwe-Kuska, and W. Römisch. Scenario reduction in stochastic programming. An approach using probability metrics. Mathematical Programming, 95(3, Ser. A):493-511, 2003.

[3] R. Fourer, D. M. Gay, and B. W. Kernighan. AMPL: A Modeling Language for Mathematical Programming. Duxbury Press / Brooks/Cole Publishing Company, 2002.

[4] H. Heitsch and W. Römisch. Generation of multivariate scenario trees to model stochasticity in power management. IEEE St. Petersburg Power Tech 2005 (to appear), 2005.

[5] M. Hollander and A. Wolfe. Nonparametric statistical inference. John Wiley \& Sons, New York, 1973.

[6] J. Nolan. stable.exe, 2004. A program to fit and simulate stable laws (http://academic2.american.edu/jpnolan/stable/stable.html for further details).

[7] G. Ch. Pflug. Scenario tree generation for multiperiod financial optimization by optimal discretization. Mathematical Programming, 89(2, Ser. B):251-271, 2001.

[8] S. Rachev and S. Mittnik. Stable Paretian Models in Finance. John Wiley \& Sons, New York, 2000.

[9] R. T. Rockafellar and S. Uryasev. Optimization of Conditional Value-at-Risk. The Journal of Risk, 2(3):21-41, 2000.

[10] A. Ruszczynski and A. Shapiro, editors. Stochastic programming, volume 10 of Handbooks in Operations Research and Management Science. Elsevier Science B.V., Amsterdam, 2003. 